\title{
SURGICAL TREATMENT OF FULL-THICKNESS MACULAR HOLES USING AUTOLOGOUS SERUM
}

\author{
JOHN A. WELLS and ZDENEK J. GREGOR \\ London
}

\begin{abstract}
SUMMARY
Background: Full-thickness macular holes (FTMH) are an important cause of visual loss in older patients. Recognition of tangential vitreofoveal traction as the mechanism of hole formation has led to the use of vitrectomy and gas tamponade as a treatment for macular holes. The use of growth factors as an adjunctive therapy to promote chorioretinal adhesion and hole closure has also been advocated.

Methods: Forty-four consecutive patients undergoing vitrectomy, posterior hyaloid removal, gas injection and installation of autologous serum for FTMH were studied prospectively. Patients' age, sex, duration of symptoms, best corrected pre- and post-operative Snellen visual acuities, best corrected acuity in the fellow eye, stage of macular hole, post-operative closure or persistence of the macular hole, and intraand post-operative complications were recorded.

Results: Of 43 patients with adequate follow-up (minimum 6 weeks, mean 6.1 months) anatomical closure of the macular hole was achieved in 29 eyes $(67 \%)$. The average visual improvement in these eyes was 2.7 lines. Twenty eyes $(69 \%)$ with closed holes had visual acuity of $6 / 12$ or better $(47 \%$ of all patients undergoing surgery). Of the 14 eyes $(33 \%)$ that failed, the average loss of vision was 0.4 lines and all these eyes had vision of $6 / 36$ or worse.

Conclusions: In this series, surgical treatment of FTMH with vitrectomy, gas injection and autologous serum resulted in closure of the macular hole in two-thirds of patients and restored central vision in about one-half of patients. These results compare favourably with previously reported results of macular hole surgery. The answer to the question whether the use of autologous serum leads to a higher closure rate than surgery with gas tamponade alone requires information from randomised treatment trials, one of which is currently in progress.
\end{abstract}

Full-thickness macular holes (FTMH) are an important cause of central visual loss. Most patients are

Correspondence to: Mr Zdenek J. Gregor, FRCS, FRCOphth, Moorfields Eye Hospital, City Road, London EC1V 2PD, UK. women in their sixth or seventh decade who present with distortion of central visual field and reduction in visual acuity to the level of $6 / 36$ or worse. Typically these lesions are non-progressive once fully established. Bilateral holes are thought to occur in approximately $10-20 \%$ of patients. ${ }^{1-5}$ The seminal observations of Gass regarding the pathogenesis and classification of FTMH $^{1,2}$ sparked a number of investigations into the efficacy of treatment with vitrectomy and gas tamponade. In 1991, Kelly and Wendel $^{6}$ reported closure of macular holes with vitrectomy and $\mathrm{SF}_{6}$ gas tamponade in $58 \%$ of their patients. It appeared that such closure is associated with cellular proliferation and, therefore, the use of promoters of the local healing response, such as growth factors, was proposed by Glaser et al. ${ }^{7}$ They reported the use of transforming growth factor beta-2 (TGF- $\beta_{2}$ ) combined with vitrectomy in an effort to improve anatomical closure rates. ${ }^{7}$ Because TGF- $\beta$ is not readily available and is expensive, autologous serum has been suggested as an alternative source of the growth factors that might promote chorioretinal adhesion and improve surgical success. ${ }^{8}$ This paper reports a series of 44 consecutive patients undergoing vitrectomy, instillation of autologous serum and gas tamponade as treatment for FTMH.

\section{Patient Selection}

Forty-four consecutive patients operated on between January 1992 and July 1993 for stage 2, 3 or 4 FTMH were studied prospectively. Patients selected for surgery had visual acuity of $6 / 12$ or worse preoperatively and were thought to be capable of performing the required post-operative face-down posturing.

\section{Clinical Evaluation}

All patients were examined by one of us and had best corrected Snellen visual acuity recorded. Dilated fundus examination was performed with indirect 
ophthalmoscopy and slit lamp examination with a 78 or 90 dioptre lens and a Goldmann fundus contact lens if uncertainty regarding the stage of the FTMH existed. The diagnosis of a FTMH was based on the presence of symptoms of distortion, central visual loss or reading difficulty, combined with the characteristic changes of macular hole formation as described by Gass. ${ }^{1,2}$ The Watzke-Allen sign of an interruption in a thin beam of light directed across the macular hole was used to assist in the diagnosis. ${ }^{9}$ All patients had colour photographs and fluorescein angiography pre-operatively. Patients were also evaluated for the presence of any general medical conditions that might prevent them from performing the 2 weeks of face-down posturing required postoperatively, and received extensive counselling on the importance of this to the success of the operation.

\section{Surgical Technique}

A three-port pars plana vitrectomy using standard techniques was performed in all patients. After removal of the core vitreous gel the vitreous cutter was switched to aspiration at $300 \mathrm{mmHg}$ and active suction over the optic disc was performed with simultaneous retraction of the cutter. In this manner the attached posterior vitreous cortex was identified in all eyes and was peeled from the retina, except in stage 4 eyes which already had spontaneous posterior vitreous separation.

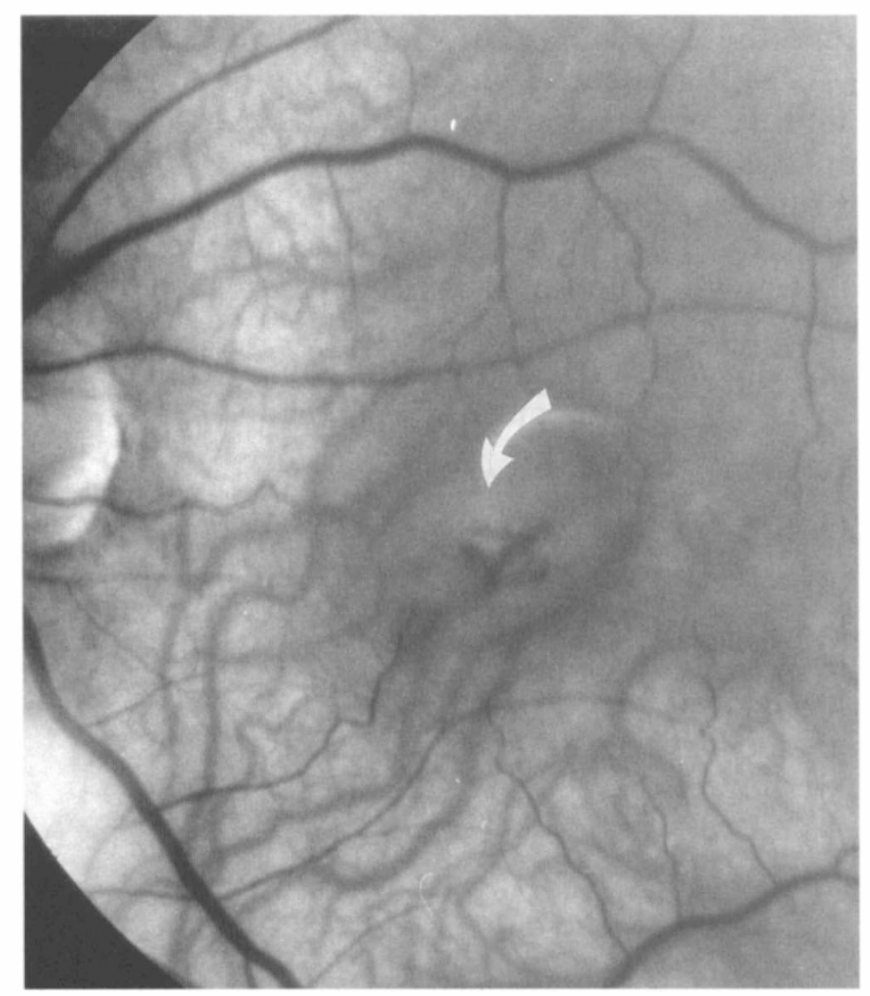

(a)
The posterior vitreous cortex was then removed with the vitreous cutter. Scleral plugs were inserted into the vitrectomy entry ports and a careful peripheral retinal examination was performed with indirect ophthalmoscopy and scleral indentation through $360^{\circ}$. Any retinal breaks detected were treated with cryopexy or laser photocoagulation. A complete fluidair exchange was then performed and the macular hole was meticulously dried with a 33 gauge extrusion cannula and active aspiration. After waiting for 10 minutes any reaccumulated fluid was removed from the retinal surface with the extrusion needle.

Autologous whole blood was obtained under sterile conditions by venepuncture and centrifuged for 5 minutes at $4000 \mathrm{rpm}$. The serum was then aspirated into a syringe and several drops instilled over the macula. The serum was allowed to stand for 5-10 minutes and then most was removed by passive aspiration over the optic disc. This left a thin residual layer of serum overlying the macula. An air- $\mathrm{C}_{3} \mathrm{~F}_{8}$ $16 \%$ gas exchange was then performed in all eyes except 4 , in which $30 \%$ sulphur hexafluoride $\left(\mathrm{SF}_{6}\right)$ gas was used as a tamponading agent. Post-operatively, the patient remained supine until sufficiently awake to assume the face-down posture required for the next 2 weeks.

\section{Post-operative Evaluation}

Patients were examined at 1 day, 2 weeks, 6 weeks,

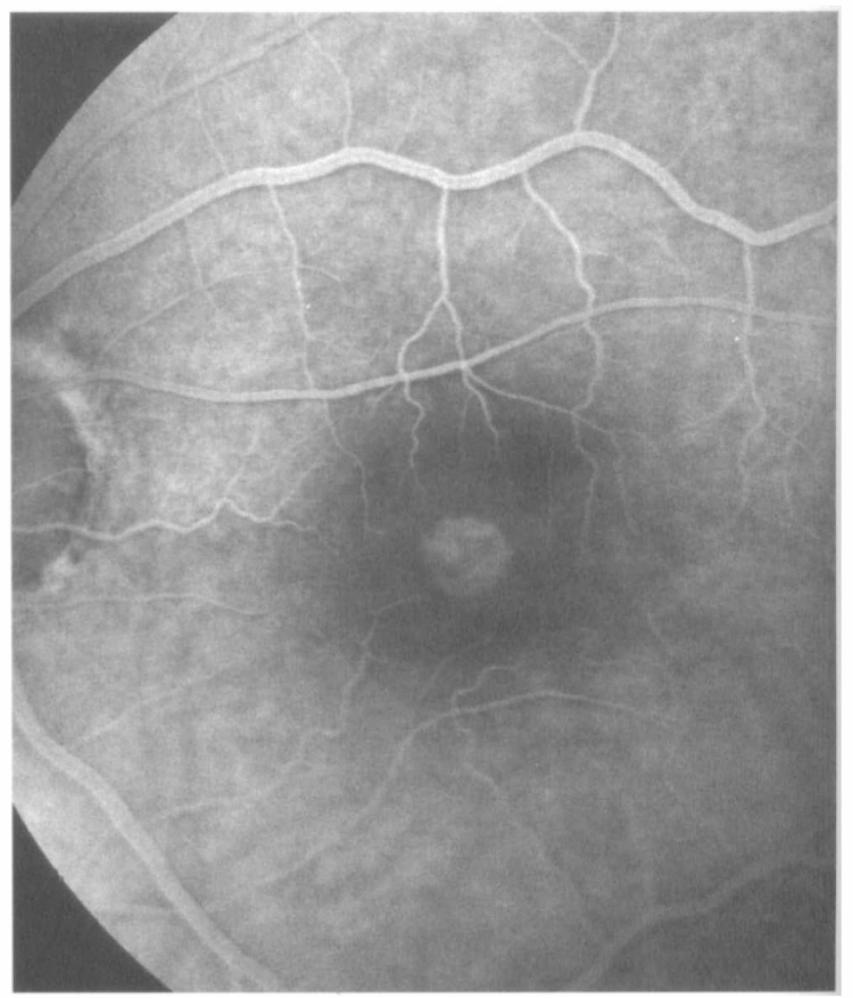

(b)

Fig. 1. (a) Red-free photograph of the case presented, showing a stage 3 FTMH with the neuroretinal defect and the cuff of subretinal fluid (arrow) clearly visible. (b) Fluorescein angiogram with hyperfluorescence delineating the margins of the retinal defect. 


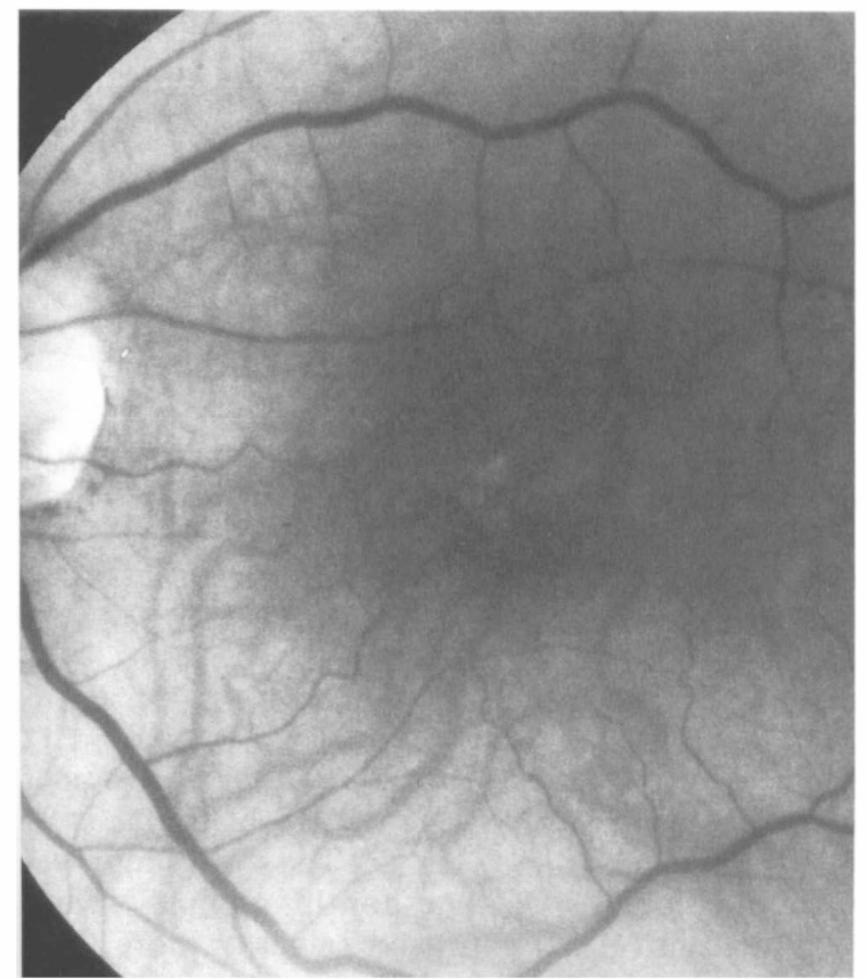

(a)

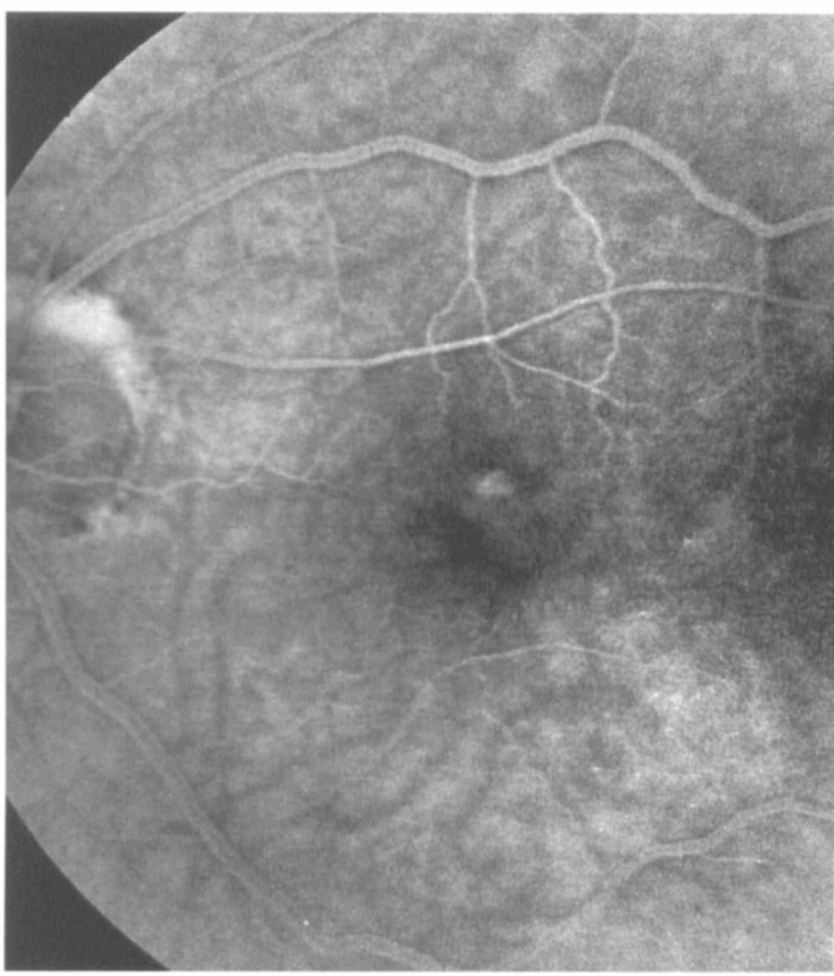

(b)

Fig. 2. (a) A red-free photograph of the same case as Fig. 1 taken 3 months post-operatively, demonstrating closure of the macular hole. (b) The post-operative fluorescein angiogram shows very faint irregular hyperfluorescence that corresponds to the depigmentation of the retinal pigment epithelium seen clinically in contrast to the round, regular hyperfluorescence seen pre-operatively.

3 months, 6 months, and thereafter 6 monthly. Best corrected Snellen visual acuity, intraocular pressure, and anatomical status of the retina and macula were evaluated in the same fashion as pre-operatively. The macular hole was considered closed if it was no longer visible or if the edges of the hole were visible but flat and without subretinal fluid surrounding the edges and the Watzke-Allen test was negative. Reoperation was offered if initial surgery failed to close the macular hole.

\section{CASE PRESENTATION}

A 72-year-old woman was first seen with a 9 month history of distortion of her right central vision and a 4 month history of distortion of her left central vision. She was in excellent health and took no medications. There was no prior history of any ocular disease.

Examination on 30 August 1994 revealed best corrected Snellen visual acuity of 6/24 in each eye. The anterior segments and intraocular pressures were normal. Fundus examination disclosed bilateral stage 3 macular holes with opercula and surrounding subretinal fluid. Fluorescein angiography confirmed these findings (Fig. 1).

After extensive discussion with the patient regarding the relative risks and benefits of surgery and the importance of the 2 weeks of post-operative posi- tioning, she agreed to undergo surgery on her left eye. On 10 October, she underwent pars plana vitrectomy, fluid-air exchange, instillation of autologous serum, and air- $16 \% \mathrm{C}_{3} \mathrm{~F}_{8}$ exchange. Postoperatively she maintained the face-down posturing as required.

The intraocular gas resorbed by the sixth postoperative week and the mascular hole was undetectable but for a mild retinal pigment epithelial depigmentation (Fig. 2). Three months post-operatively the best corrected Snellen acuity was 6/12.

\section{RESULTS}

Minimum follow-up was considered to be 6 weeks post-operative, by which time the intraocular gas had largely resorbed and allowed accurate assessment of the anatomical status of the macula and visual acuity. Adequate follow-up was available for 43 patients (one patient died within 6 weeks of surgery from undiagnosed metastatic ovarian carcinoma). Three patients had two operations.

The 44 patients included 35 women $(79.5 \%)$ and 9 men $(20.5 \%)$. The mean age was 66.3 years (range 44-82 years). The mean age by sex was 64.1 years (range 44-74 years) for women and 68.6 years (range 47-82 years) for men. The average duration of symptoms was 6 months (range 1-18 months). All eyes had an idiopathic macular hole except 4 that 
Table I. Patient summary

\begin{tabular}{llll}
\hline Mean age & 66.3 years & (range $44-82$ years) \\
Sex & 35 female & $(80 \%)$ \\
& 9 male & $(20 \%)$ & \\
Mean duration of symptoms & 6.1 months & (range & $1-18$ months) \\
Stage of FTMH & Stage 2 & $14 \%$ & $(6 / 43)$ \\
& Stage 3 & $77 \%$ & $(34 / 43)$ \\
Bilateral holes & Stage 4 & $9 \%$ & $(4 / 43)$ \\
\hline
\end{tabular}

FTMH, full-thickness macular hole.

Table II. Pre- and post-operative Snellen visual acuities for all eyes $(n=43)$

\begin{tabular}{lccr}
\hline & \multicolumn{3}{c}{ Visual acuity } \\
\cline { 2 - 4 } & Pre-operative & Post-operative & Fellow eye \\
\hline $6 / 12$ or better & 0 & $20 / 43(47 \%)$ & $26 / 43(63 \%)$ \\
$6 / 18-6 / 36$ & $21 / 43(49 \%)$ & $11 / 43(25 \%)$ & $5 / 43(12 \%)$ \\
$6 / 60$ or worse & $22 / 43(51 \%)$ & $12 / 43(28 \%)$ & $10 / 43(24 \%)$ \\
\hline
\end{tabular}

had myopia of greater than 6 dioptres. There were $6(14 \%)$ stage 2 holes, $34(77 \%)$ stage 3 holes, and 4 (9\%) stage 4 holes (Table I). Thirteen patients $(29.5 \%)$ had bilateral macular holes and in each of these cases the eye with the shortest duration of symptoms was chosen for surgery.

Pre-operative visual acuity was less than $6 / 18$ in all cases. Forty-nine per cent (21/43) of eyes had a visual acuity of $6 / 18-6 / 36$, and $51 \%$ (22/43 eyes) had a visual acuity of $6 / 60$ or less. The fellow eyes had vision of $6 / 12$ or better in $63 \%(26 / 43)$ of eyes, $6 / 18$ $6 / 36$ in $12 \%$ (5/43 eyes) and $6 / 60$ or less in $24 \%(10 /$ 43 eyes). Of the 10 fellow eyes with vision of $6 / 60$ or less, 7 had a FTMH. A summary of the pre- and postoperative visual acuity results is given in Table II.

\section{Anatomical Results}

Successful closure of the macular hole was achieved in $67 \%(29 / 43)$ of eyes. Analysis of the effect of the pre-operative stage and closure rates showed that $100 \%(6 / 6)$ of stage 2 holes, $64 \%(21 / 33)$ of stage 3 holes, and $50 \%(2 / 4)$ of stage 4 holes were closed. Closure rates also varied according to the duration of symptoms, with $70 \%(28 / 42)$ closure in eyes with symptoms for less than 1 year, $80 \%$ (23/29) closure in eyes with symptoms for less than 6 months, and $87 \%$

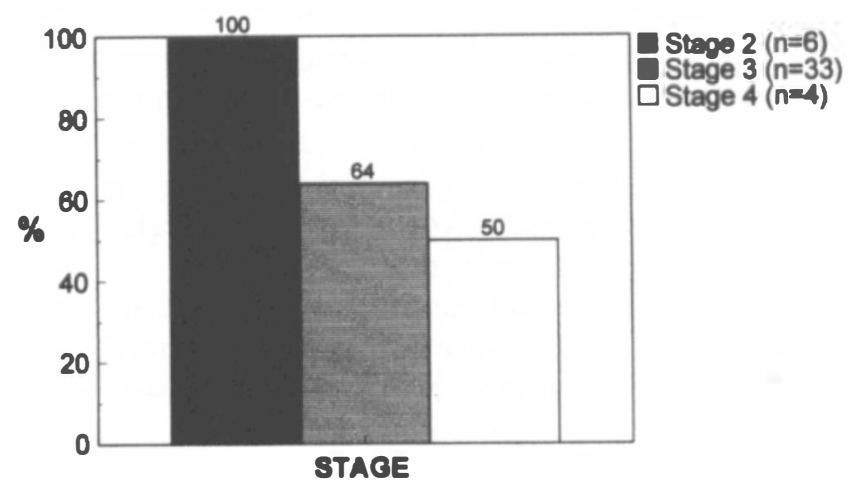

Fig. 3. Bar graph illustrating closure rates by macular hole stage.

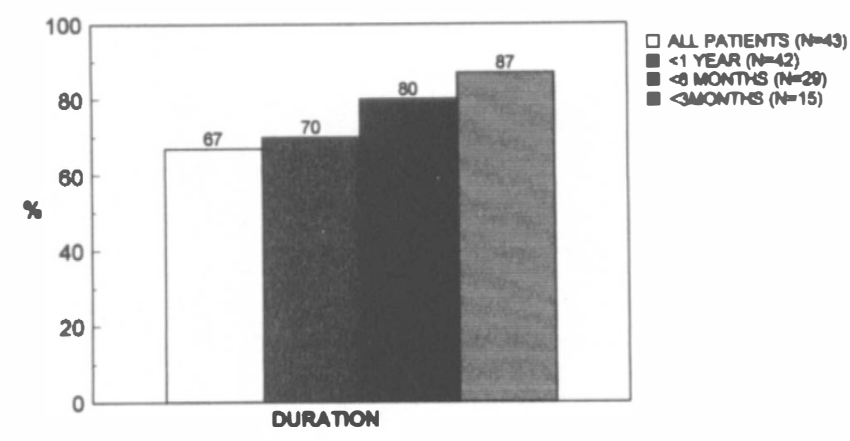

Fig. 4. Bar graph demonstrating closure rates by duration of symptoms.

$(13 / 15)$ closure in eyes with symptoms for less than 3 months (Table III; Figs. 3, 4).

\section{Visual Results}

Of the 29 closed holes, $69 \%$ (20/29) attained vision of $6 / 12$ or better, $27.5 \%(8 / 29)$ saw $6 / 18-6 / 36$, and $3.5 \%$ (1/29) saw 6/60 or less (Fig. 5). The mean improvement in vision was +2.7 lines for this group. Seventytwo per cent (21/29) improved 2 lines or more, $24 \%$ (7/29) were unchanged ( \pm 1 line), and $4 \%(1 / 29)$ lost two lines. In analysing the visual results of the subgroups with the highest closure rates, the eyes with symptoms for less than 3 months had somewhat better post-operative vision, with $77 \%$ achieving $6 / 12$ or better and a mean gain of 3 lines. The eyes with stage 2 holes pre-operatively also had slightly better outcomes, with $83 \%(5 / 6)$ achieving $6 / 12$ or better.

Of the 14 eyes with persistently open macular holes, all had vision of $6 / 36$ or worse, with $79 \% 6 / 60$ or worse (11/14 eyes) and 21\% (3/14 eyes) having $6 / 36$ vision. The average loss of vision was 0.4 lines. One patient lost 2 lines of vision, and vision in the other $93 \%$ of this group was unchanged, with \pm 1 line different compared with pre-operative vision (Table IV).

Taking the entire group as a whole, $47 \%$ (20/43) of

Table III. Macular hole closure rates

\begin{tabular}{lc} 
All eyes & $29 / 43(67 \%)$ \\
Stage & \\
2 & $6 / 6(100 \%)$ \\
3 & $21 / 33(64 \%)$ \\
4 & $2 / 4(50 \%)$ \\
Duration & \\
$\quad>1$ year & $0 / 1$ \\
$<1$ year & $28 / 42(70 \%)$ \\
$<6$ months & $23 / 29(80 \%)$ \\
$<3$ months & $13 / 15(87 \%)$ \\
\hline
\end{tabular}

Table IV. Visual change by Snellen lines

\begin{tabular}{lccc}
\hline & $\begin{array}{c}\text { All eyes } \\
(n=43)\end{array}$ & $\begin{array}{c}\text { Closed holes } \\
(n=29)\end{array}$ & $\begin{array}{c}\text { Open holes } \\
(n=14)\end{array}$ \\
\hline + 2 lines or more & $21 / 43(49 \%)$ & $21 / 29(72 \%)$ & $(0)$ \\
\pm 1 line & $20 / 43(47 \%)$ & $7 / 29(24 \%)$ & $13 / 14(93 \%)$ \\
- 2 lines or more & $2 / 43(4 \%)$ & $1 / 29(4 \%)$ & $1 / 14(7 \%)$ \\
$\begin{array}{l}\text { Mean gain/loss of } \\
\text { Snellen lines }\end{array}$ & +1.7 & +2.7 & -0.4 \\
\hline
\end{tabular}




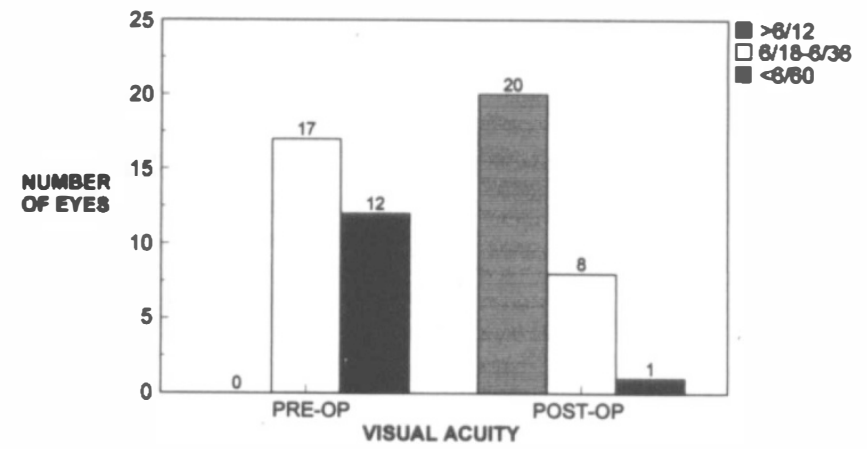

Fig. 5. Pre-operative and post-operative visual acuities of patients with closed macular holes.

eyes had a visual acuity of $6 / 12$ or better, $25 \%(11 / 43$ eyes) of $6 / 18-6 / 36$, and $28 \%$ (12/43 eyes) of $6 / 60$ or worse following surgery. Forty-nine per cent (21/43) of eyes gained 2 lines or more, $47 \%$ (20/43 eyes) were \pm 1 line, and $4 \%$ (2/43 eyes) lost 2 or more lines.

Complications of surgery, either intra- or postoperative, were noted in $23 \%(10 / 43)$ of eyes. Five eyes developed retinal tears (4 intraoperatively and 1 six weeks post-operatively) and 2 developed retinal detachments requiring further surgery. Two patients developed peripheral visual field defects noted after resorption of the intraocular gas. The cause of these defects could not be determined despite thorough retinal and neuro-ophthalmological evaluation. One aphakic patient developed a hyphaema and increased intraocular pressure post-operatively that responded well to further surgery, and ultimately achieved a visual acuity of $6 / 6$. Of all the patients experiencing complications, none lost 2 or more lines of vision as a result of the complication (Table V).

\section{DISCUSSION}

In 1988, Gass proposed a classification of the stages of the development of idiopathic macular holes and suggested that focal tangential traction on the fovea by adherent posterior cortical vitreous was the main mechanism of hole formation. He also suggested that vitrectomy and the removal of the attached posterior hyaloid could relieve the vitreofoveal traction and prevent the formation of a FTMH. ${ }^{1,2}$ However, a recently reported randomised trial of surgery for stage 1 macular holes in the fellow eye of patients with FTMH failed to demonstrate a benefit of surgery. ${ }^{10}$

In 1991, Kelly and Wendel ${ }^{6}$ reported the first series of patients undergoing vitrectomy, removal of the posterior cortical vitreous, peeling of epiretinal membranes when present, and gas tamponade with $\mathrm{SF}_{6}$ for stage 3 and $4 \mathrm{FTMH}$. They obtained a closure rate of 58\% (30/52 eyes) and an improvement of two or more lines of vision in $73 \%$ of the eyes that had closed holes (22/30 eyes) or $42 \%$ (22/52 eyes) of the entire group. ${ }^{6}$ Though not reported by the authors,
Table V. Complications

\begin{tabular}{ll}
\hline Retinal tears & 5 \\
Intra-operative & 4 \\
Post-operative & 1 \\
Retinal detachment & 2 \\
Visual field defects & 2 \\
Hyphaema & 1 \\
\hline
\end{tabular}

review of their data shows that $36 \%(11 / 30)$ of the eyes with closed holes achieved $6 / 12$ or better visual acuity ( $21 \%$ of the entire group). Subsequently these same surgeons have presented two updated nonrandomized series of patients, the first reporting anatomical closure in $73 \%$ of 170 cases $^{11}$ and the most recent reporting an $89 \%$ closure rate in 276 consecutive cases (oral presentation, American Academy of Ophthalmology Annual Meeting, November 1994). This suggests that with further experience the anatomical success rates are greater. Disappointingly, however, these authors were still able to record visual acuity of $6 / 12$ or better in only $40 \%$ of the eyes with closed holes and symptoms of less than 1 year's duration.

In 1992 Glaser et al. ${ }^{7}$ reported the use of bovine transforming growth factor-beta (TGF- $\beta_{2}$ ) as an adjunct to vitrectomy and gas tamponade for FTMH. ${ }^{7}$ The rationale for the use of TGF- $\beta_{2}$ is that it has been shown to promote chorioretinal adhesion by stimulating collagen and proteoglycan synthesis as well as cellular proliferation and migration involved in wound healing. ${ }^{8,12}$ In this study three different concentrations of TGF- $\beta_{2}$ were applied to the macular hole with or without hyaluronic acid. There was a $100 \%$ (23/23 eyes) closure rate in the two groups receiving 330 and $1330 \mathrm{ng}$ of TGF- $\beta_{2}$ without hyaluronic acid, and $61 \%$ of these eyes showed an improvement in visual acuity of two lines or more. ${ }^{10}$ The other four groups in this study fared much worse, with only $38 \%$ (14/37 eyes) of holes closing.

Because TGF- $\beta$ is not readily available and is expensive, autologous serum was proposed by Liggett $e t$ al. $^{12}$ as an easily obtainable and inexpensive source of TGF- $\beta$ and a variety of other growth factors that could theoretically have the same positive effects on wound healing and formation of a chorioretinal adhesion. In an experimental rabbit model of retinal holes autologous serum has been shown to produce a chorioretinal adhesion compared with controls not treated with serum. ${ }^{8}$ Because serum may stimulate fibrocellular proliferation and chorioretinal adhesion without concomitant photoreceptor destruction, it would seem to be of value in macular holes, where preservation of viable photoreceptors is critical to good visual outcomes. Given this background we undertook our own study of surgery for FTMH using autologous serum. The results of our study show a closure rate of $67 \%$ that is comparable to the previous reports. Our visual results are also 
favourably comparable: $69 \%$ (20/29 eyes) of the eyes with closed holes achieved 6/12 vision or better and $72 \%$ (21/29 eyes) gained two or more Snellen lines of acuity. These results are similar to the $73 \%$ and $61 \%$ rates of improvement by two Snellen lines or more reported by Kelly and Wendel ${ }^{6}$ and Glaser et al. ${ }^{7}$ respectively. When considering our group as a whole, however, only $46 \%$ achieved $6 / 12$ or better, a figure that is again very similar to the $40 \%$ reported by Wendel at the American Academy of Ophthalmology in 1994. This discrepancy between closure rates and visual outcomes is the most disappointing aspect of the experience with macular hole surgery to date.

The reasons for poor vision in macular holes was initially assumed to be the loss of a small segment of neurosensory retina ('foveal operculum') with surrounding cystoid oedema and photoreceptor degeneration. $^{1-5,14}$ However, clinicopathological examination of eyes that have had successful surgery for idiopathic macular holes found a small defect in the external limiting membrane measuring 16-50 $\mu \mathrm{m}$ in diameter with a central full-thickness plug of glial tissue thought to be Müller cells. The photoreceptors and the retinal pigment epithelium outside the defect were found to be normal in appearance. There was no evidence of inflammation or cystoid macular oedema in these successfully treated eyes. ${ }^{15,16}$ The small size of the defects in the neurosensory retina after vitrectomy suggests that there is no loss of tissue during hole formation but rather that there is centrifugal traction on the fovea by the attached vitreous cortex creating a dehiscence in the fovea, as suggested previously by others. ${ }^{4}$ Therefore, good visual acuity would be possible if the vitreous traction were relieved and allowed the edges of the foveal dehiscence, and therefore the intact photoreceptors, to reappose. Whether the same mechanisms pertain in myopic eyes with macular holes is debatable, and the small number of such eyes in our study does not allow us to draw any useful conclusions from this group of patients.

Failure of some eyes to improve visual acuity may be associated with photoreceptor degeneration, and pre-operative identification of such patients might allow selection of those cases likely to recover good vision. Our subgroup of patients who were operated within 3 months of symptom onset showed a higher closure rate $(87 \%)$ and a slightly better visual outcome ( $77 \%$ of closed holes $6 / 12$ or better; average improvement of three Snellen lines) than the group as a whole, supporting the concept that less photoreceptor degeneration may have occurred in these eyes. Others have also noted a better outcome in eyes that have been affected for less than 1 year. ${ }^{11}$ Also, we found a $100 \%$ closure rate in eyes with stage 2 holes - an earlier stage that might be expected to have less photoreceptor damage. Indeed,
$83 \%$ (5/6 eyes) of stage 2 eyes achieved $6 / 12$ or better as opposed to $69 \%$ of the eyes with closed holes of all stages and $47 \%$ of the entire group. We acknowledge that the number of eyes in this study is relatively small, particularly with regard to the more advanced (stage 4) holes. Because the prognosis seems poorer for long-standing FTMH, we usually do not recommend surgery for patients who have been affected for more than 1 year.

Some authors have questioned the appropriateness of such an invasive procedure, with its attendant risks and high rates of post-operative cataract formation, that also requires very demanding and uncomfortable post-operative posturing, for a condition that is usually unilateral, affects only central vision, and is non-progressive. ${ }^{3,17}$ For this reason, we looked at the visual acuity in the fellow eyes of our patients. A significant number (10/41 eyes, or $24 \%)$ had vision of less than $6 / 60$ in the fellow eye, and only $63 \%$ (26/41 eyes) had vision of $6 / 12$ or better. In fact, in $30 \%$ (13/44 eyes) of cases the fellow eye had vision worse than or equal to the eye with the FTMH pre-operatively. Six of these cases had bilateral macular holes, and the causes of poor vision in the other fellow eyes included glaucoma, amblyopia and macular degeneration. Clearly, in our group a significant number of patients were seriously disabled by the occurrence of a FTMH in their better eye, and surgery could potentially offer them substantial benefit. While we do not deny that vitreous surgery carries significant risk, only two patients in the entire series $(4.6 \%)$ experienced a loss of acuity of two or more Snellen lines.

In summary, our study shows that using the operative methods described, about two-thirds of patients with full-thickness macular holes can be expected to have closure of the hole and about half can expect an improvement in vision to the level of $6 / 12$ or better. While complications were not insignificant $(23 \%)$, less than $5 \%$ resulted in visual loss. In this paper we do not address the major problem of post-operative nuclear sclerosis, which is likely to occur in a large proportion of these patients, as it is the subject of a separate communication. However, for a condition that was previously regarded as untreatable, surgery for FTMH offers a reasonable and relatively safe chance for the recovery of central vision.

Presented in part at the Congress of the Royal College of Ophthalmologists, 1995. Supported by a Grant from the Association for the Guide Dogs for the Blind.

Key words: Macular hole, Vitrectomy, Autologous serum.

\section{REFERENCES}

1. Gass JDM. Idiopathic senile macular hole: its early stages and pathogenesis. Arch Ophthalmol 1988;106: 629-39. 
2. Johnson RN, Gass JDM. Idiopathic senile macular holes: observations, stages of formation, and implications for surgical intervention. Ophthalmology 1988;95: 917-24.

3. Guyer DR, de Bustros S, Diener-West M, Fine SL. Observations on patients with idiopathic macular holes and cysts. Arch Ophthalmol 1992;110:1264-8.

4. Smith RG, Hardman Lea SJ, Galloway NR. Visual performance in idiopathic macular holes. Eye 1990;4: 190-4.

5. Acosta F, Lashkari K, Reynaud X, Jalkh AE, van de Velde F, Chedid N. Characterization of functional changes in macular holes and cysts. Ophthalmology 1991;98:1820-3.

6. Kelly NE, Wendel RT. Vitreous surgery for idiopathic macular holes: results of a pilot study. Arch Ophthalmol 1991;109:654-9.

7. Glaser BM, Michels RG, Kupperman BD, Sjaarda RN, Pena RA. Transforming growth factor- $\beta_{2}$ for the treatment of full thickness macular holes: a prospective randomized study. Ophthalmology 1992;99:1162-73.

8. Christmas NJ, Skolik SA, Howard MA, Saito Y, Barnstable CJ, Liggett PE. Treatment of retinal breaks with autologous serum in an experimental model. Ophthalmology 1995;102:263-71.

9. Watzke RC, Allen L. Subjective slit-beam sign for macular disease. Am J Ophthalmol 1969;68:449-53.

10. de Bustros S, and The Vitrectomy for Prevention of
Macular Hole Study Group. Vitrectomy for prevention of macular hole: results of a randomized multicenter clinical trial. Ophthalmology 1994;101:1055-60.

11. Wendel RT, Patel AC, Kelly NE, Salzano TC, Wells JW, Novack GD. Vitreous surgery for macular holes. Ophthalmology 1993;100:1671-6.

12. Smiddy WE, Glaser BM, Green WR, Connor TB, Roberts AB, Lucas R, Sporn MB. Transforming growth factors beta: a biologic chorioretinal glue. Arch Ophthalmol 1989;107:577-80.

13. Liggett PE, Skolok SA, Hario B, Saito Y, Alfaro V, Mieler W. Human autologous serum for the treatment of full-thickness macular holes: a preliminary study. Ophthalmology 1995;102:1071-6.

14. Guyer DR, Green WR, de Bustros S, Fine SL. Histopathologic features of idiopathic macular holes and cysts. Ophthalmology 1990;97:1045-51.

15. Funata M, Wendel RT, De La Cruz Z, Green WR. Clinicopathologic study of bilateral macular holes treated with pars plana vitrectomy and gas tamponade. Retina 1992;12:289-98.

16. Madreperla SA, Geiger GL, Funata M, de la Cruz Z, Green WR. Clinicopathologic correlation of a macular hole treated by cortical vitreous peeling and gas tamponade. Ophthalmology 1994;101:682-6.

17. Fine SL. Vitreous surgery for macular hole in perspective: is there an indication? Arch Ophthalmol 1991;109:635-6. 